\title{
Role of p53-Mediated Apoptosis in Limiting Metastasis
}

\author{
Sandra Anne Hegeman, Haya Ghannouma, \& Lei Zhou \\ University of Florida
}

Faculty mentor: Lei Zhou, Department of Molecular Genetics and Microbiology

\begin{abstract}
p53 is a transcription factor known to play important roles in limiting tumorigenesis, including controlling the induction of pro-apoptotic genes and apoptosis in response to oncogenic stress. Using ChIP-Seq and RNA-Seq, our lab has previously identified p53 binding sites potentially responsible for p53-mediated induction of pro-apoptotic genes following DNA damage. Using CRISPR Cas9, we have generated fly strains with deletions or mutations within the p53 binding motif located in the experimentally verified p53 binding site. This deletion blocked DNA damage induced apoptosis. To study the functionality of the motif in limiting tumorigenesis, we introduced the deletion into a genetic tumorigenesis and metastasis model, where the tumor-suppressor cell polarity gene scribble was knocked down via shRNAi in the nonessential tissue compound eye and introduced together with strong oncogenic mutation that can lead to neoplasia and metastasis. We developed a scoring scale to quantify the severity of neoplasia and metastasis, where 0 indicates a wild type phenotype and 5 indicates the most severe metastatic phenotype. Results show that the p53 binding site knockout predominantly displayed a score of 5 (39\%). However, our preliminary results also suggested that the severity of this model may also be subjected to genetic background besides the p53 binding motif.
\end{abstract}

\section{Introduction}

The protein p53 is known to have a multitude of varying functions, though not all entirely understood. This transcription factor has been shown to act as a tumor suppressor through a number of mechanisms, including the induction of pro-apoptotic genes that function to maintain genome integrity by destroying oncogenic cells (Amundson, Myers, Fornace, 1998). Growing evidence suggests that the loss of functional p53 fosters the acquisition of additional mutations that promote a tumorigenic metastatic environment (Powell, Piwnica-Worms, \& PiwnicaWorms, 2014). p53 plays a role in regulating cell cycles, and mutations of p53 are linked to Myc amplification and overexpression seen in many cases of cancer (Mishra, Brat, \& Verma, 2014). Epigenetic dysfunction in the p53 promoter region causes p53 suppression and can result in multiple types of cancer, including breast, adrenal, head, and neck (Mishra, Brat, \& Verma, 2014). Additionally, a large majority of those who suffer from cancerous tumors have been found to have a mutated, dysfunctional form of p53 present within the tumors (Duffy, Synnott, Crown, 2017). Because metastasis is the primary cause of death in a majority of cancer cases 
(Seyfried \& Huysentruyt, 2013), understanding underlying mechanisms of the process may be vital.

A 33 kbp highly conserved region within the Drosophila genome known as the irradiationresponsive enhancer region (IRER) has been proven to play a role in tumor suppression through the induction of three pro-apoptotic genes known as reaper, hid, and sickle (Zhang et al., 2014). A robust induction of these genes has been shown following irradiation in the sensitive stage of embryonic development (stages 9-11) of Drosophila. At embryonic development stage 12, the epigenetic status of the IRER shifts to a closed configuration and irradiation-induced apoptosis is blocked (Zhang et al., 2008).

Chip-seq and RNA-seq methods in our previous studies, have allowed us to identify a p53 binding site within the IRER (Zhou lab, in preparation), although the exact functionality and impact of this binding site on tumorigenesis and metastasis is not yet fully understood. In this study, we aim to determine the effects of mutating the p53 binding site on tumorigenesis and metastasis in a tumor-susceptible Drosophila mode. The animals will be monitored and assessed for the effects of the mutation on metastasis and tissue neoplasia to allow us to expand our understanding of the relationship between p53, neoplasia, metastasis, and ultimately cancer.

Clustered regularly interspaced short palindromic repeats (CRISPR) is a genome editing tool used to manipulate an organism's genetic code (DiCiaccio, Acuna, Ghannouma, et al, unpublished data). A guide RNA (gRNA) was designed to target a complementary DNA sequence designating the p53 binding site within the IRER (Zhao et al, 2017). Using CRISPRCas9 technology, our group has previously generated fly lines with deletions or mutations within the p53 binding motif (R8-1) and a wild-type mutant (R15-1) (Figure 1) that may disrupt the induction of pro-apoptotic genes located within the IRER, such as reaper and hid.

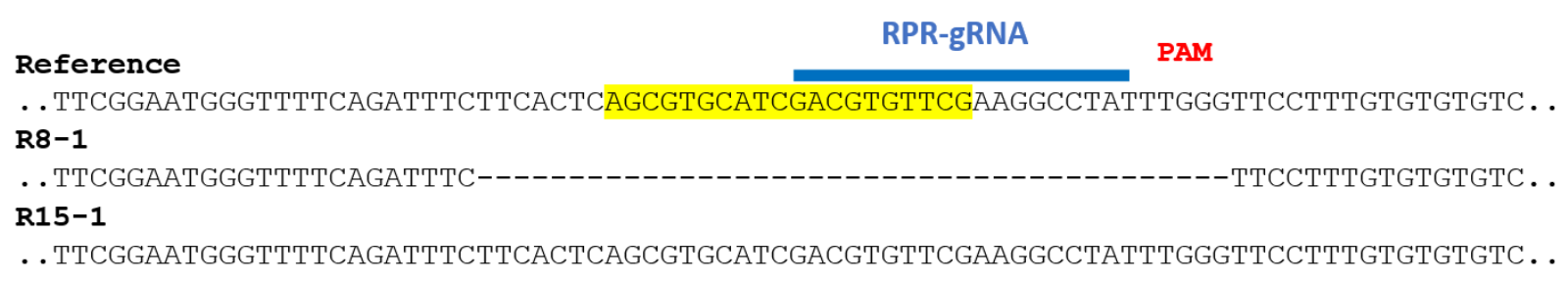

Figure 1. CRISPR Cas9 generated fly stocks. R8-1 has a 41 bp mutation that deletes the entire p53 binding site. R15-1 has undergone CRISPR gene editing without mutations to the binding site. 


\section{Methods}

\section{Verification of CRISPR-Cas9 Generated Fly Lines}

Primers were designed to flank the regions upstream and downstream of the gRNA sequence. The sequence was amplified using Polymerase chain reaction (PCR) and then processed for Sanger sequencing to identify mutations to the p53 binding site of each CRISPR-generated fly line (Verma, Kulshrestha, Puri, 2017). Mutations to the p53 binding site motif were analyzed by aligning the nucleotide sequence of the mutants to Basic Local Alignment Search Tool (BLAST) databases, including FlyBase (Thurmond et al., 2019) and the National Center for Biotechnology Information's (NCBI) (Altschul et al., 1990).

\section{Knockdown of Scribble with shRNAi and the ey1x-Gal4/UAS System}

The scribble protein, which is functionally known to maintain cell polarity, is knocked down via short hairpin RNA interference (shRNAi) to promote a tumorigenic metastatic environment (Moore et al, 2010). In our model, Gal4, a yeast transcription activator, is expressed in the Drosophila's eyes. Once crossed with a strain containing UAS-ScribRNAi (with UAS being the Upstream Activator Sequence), Gal4 will bind and activate the gene transcription of ScribRNAi, inducing a metastatic phenotype (Busson, Pret, 2007).

\section{Drosophila Strains and Culture}

Flies were maintained in a standard corn-agar medium at $25^{\circ} \mathrm{C}$. The strains used in this study are described in Table 1.

Table 1. Fly strains and genotypes

\begin{tabular}{|l|l|l|}
\hline Strain & Genotype (Thurmond et al, 2019) & Notes \\
\hline M042 & w[1118]; UAS-scrib RNAi & recombine the scrib RNAi (from G364) with \\
& (HMS01490), & Df(IRER), FRT chr \\
& Df\{IRER $[$ [B39]:FRT80B/TM6B & \\
& Tb-24 & \\
\hline M072 & ey1x-Gal4:UAS-DsRed/Cyo;rpr & From vial R8-1X. \\
& BSKO (R8-1)/Tm3,Sb & 18403346:TTCGGAATGGGTTTTCAGATTT \\
& & C------------------------------- \\
& & TTCCTTTGTGTGTGTC:18403425 (41bp \\
& & deletion) (Tm3,Sb should go away after a few \\
& & generation 8/22) \\
\hline
\end{tabular}




\begin{tabular}{|l|l|l|}
\hline M091 & ey1x-Gal4:UAS-DsRed/Cyo;rpr & From vial R15-1X. \\
& BSKO (R15-1)/Tm3,Sb & 18403346:TTCGGAATGGGTTTTCAGATTT \\
& & CTTCACTCAGCGTGCATCGACGTGTTCG \\
& & AAGGCCTATTTGGGTTCCTTTGTGTGTGT \\
& & C:18403425 (no mutation) (Tm3,Sb should go \\
& & away after a few generation 8/22) \\
\hline G132 & ey1xgal4: UAS- & None \\
& DsRed/Cyo;Df(IRER)/T3Sb & \\
\hline
\end{tabular}

Three experimental crosses were generated with four vials per cross containing equal amounts of male and female flies allowing to propagate for 10 days. At day 10, the parents from each vial were moved. The offspring of the parental flies, or the F1 generation, was segregated into labeled vials based on their genotypes for 8 days after removal of parents. The flies were frozen in $-80^{\circ} \mathrm{C}$ until they were ready to be scored on the metastasis scoring scale.

Our first cross (1.1) was a cross between male drosophila that had a full p53 binding motif deletion, BSKO (R8-1), as well as DsRed fluorescent protein markers with the Gal4/UAS system, and female virgin drosophila that were IRER deficient with scribble knocked down (Figure 2). The purpose of DsRed is to monitor gene expression while working in conjunction with the Gal4/UAS system, which regulates transcription. We used a Leica MZ 16 F infrared microscope with the ability to identify the DsRed fluorescent marker to verify that both our parental drosophila and our F1 generation expressed DsRed. Our goal for this cross was to observe what metastasis (if any) occured in the phenotype of the F1 offspring that had both DsRed and the p53 binding motif deletion in their genotype. This served as our experimental cross.

Cross 1.1

M072 (R8-1)

$\mathrm{M} 042$

$\frac{\text { ey1 } 1 x-\text { Gal4:UAS-DSRed }}{\text { Cyo }} ; \frac{\operatorname{rpr} B S K O(R 8-1)}{\operatorname{rpr} B S K O(R 8-1)} \quad \mathrm{X} \quad w[1118] ; \frac{+}{+} ; \frac{U A S-S C r i b R N A i, d f(I R E R)[B 39]: F R T 80 B}{T m 6, T b}$

Examine F1 $\frac{\text { ey1x-Gal4:UAS-DsRed }}{+} ; \frac{\text { UAS-ScribRNAi:df(IRER })}{r p r B S K O(R 8-1)}$ for metastatic phenotype

Figure 2. Cross 1.1 between CRISPR generated R8-1 stocks with a 41 bp deletion in the p53 binding motif, and a strain containing UAS-ScribRNAi to generate a metastatic fly line. 
Our second cross (1.2) was a cross between male drosophila that had DsRed fluorescent protein markers with the Gal4/UAS system, but did not have a p53 binding motif deletion, BSKO (R15-1), and female virgin drosophila that were IRER deficient with scribble knocked down (Figure 3)

Cross 1.2

M091 (R15-1)

$\frac{\text { ey1x-Gal4:UAS-DSRed }}{\text { Cyo }} ; \frac{\operatorname{rpr} B S K O(R 15-1)}{\operatorname{rpr} B S K O(R 15-1)} \quad \mathrm{X} \quad w[1118] ; \frac{+}{+} ; \frac{\text { UAS-SCribRNAi,df }(\text { IRER })[B 39]: F R T 80 B}{T m 6, T b}$

Examine F1 $\frac{\text { ey1x-Gal4:UAS-DsRed }}{+} ; \frac{\text { UAS-ScribRNAi:df(IRER })}{r p r \text { BSKO }(R 15-1)}$ for metastatic phenotype

Figure 3. Cross 1.2 between CRISPR generated R15-1 stocks with no mutations to the binding site, and a strain containing UAS-ScribRNAi to generate a control fly line with which to compare to the metastatic fly line from cross 1.1 .

Our third cross (2) was a cross between male and female drosophila that were both heterozygous for an IRER deficiency. Our goal for this cross was to examine the offspring that were homozygous for the IRER deficiency for metastasis. We also observed the offspring that were still heterozygous for the deficiency, as they also displayed metastasis. This cross served as our control (Figure 4).

Cross 2

G132

$\frac{\text { ey } 1 x-\text { Gal4:UAS-DSRed }}{C y o} ; \frac{d f(I R E R)}{T m 3, S b} \quad \mathrm{X} \quad w[1118] ; \frac{+}{+} ; \frac{\text { UAS-SCribRNAi,df }(\text { IRER })[B 39]: F R T 80 B}{T m 6, T b}$

Examine F1 $\frac{\text { ey1x-Gal4:UAS-DsRed }}{+} ; \frac{\text { UAS-ScribRNAi:df(IRER })}{d f(I R E R)}$ for metastatic phenotype

Figure 4. Cross 2 between an IRER deficient strain, and a strain containing UAS-ScribRNAi to generate a severe metastatic phenotype in the F1 generation.

\section{Results}

\section{Ranking of Neoplasia and Metastasis}

The scale developed in-house was used to rank the severity of metastasis observed in the F1 generation as follows (Figure 5). 
0: Wild type or normal. Eye Shape found in a wild type fly. Healthy looking and complete in size.

1: Smaller/Rough eye. The eye may be smaller or rough looking but there is not extra growth or invagination happening in the eye.

2: Deformed eye. You may notice an invagination or bean shape in the eye.

3: Local neoplasia. Local neoplasia eyes are those that have tumors on the eye or extremely close to the eye area. This would be considered the black growths of any growth that does not look like eye cells even though it is located on the eye.

4: Migrating/Ectopic eye. Ectopic eyes can be labeled as those that seem like a piece of the eye has migrated to somewhere further than the eye. You might see a red or orange small tissue on the antenna or somewhere away from the eye.

5: Neoplasia and metastasis. Very obvious neoplasia that has deformed the eye and head. This will also include those that have a small black growth (tumor) somewhere other than the eye such as the antenna or mouth.

Only one of the two eyes need to have the corresponding phenotype to be labeled as so.

\section{Scoring of Metastatic Phenotype}

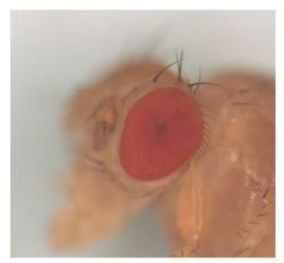

O Wild Type

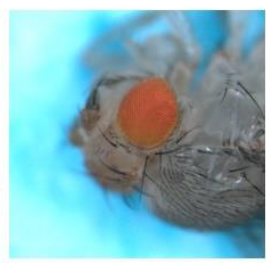

1 Small or Rough Eye

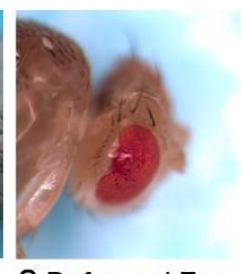

2 Deformed Eye

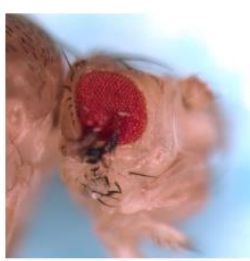

3 Local Neoplasia

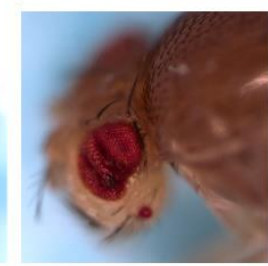

4 Migrating or Ectopic Eye

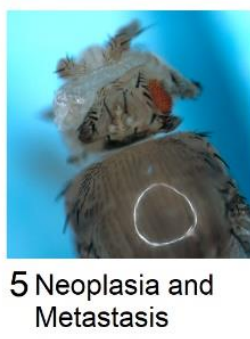

5 Neoplasia and Metastasis

Figure 5. Metastasis Scoring Guidelines

\section{Df(IRER) Leads to Increased Metastasis}

In our third cross (2) we found that out of the eight genotypes that were expressed, six had a metastatic score of zero. These six genotypes were:

1. ey1x-Gal4:DsRed/+; df(IRER)/Tm6,Tb

2. ey1x-Gal4:UAS-DsRed/+; Tm3,Sb/Tm6,Tb

3. Cyo/+ ; UAS-ScribRNAi, df(IRER)/df(IRER)

4. Cyo/+ ; UAS-ScribRNAi, df(IRER)/Tm3,Sb

5. Cyo/+ ; df(IRER)/Tm6,Tb

6. $\mathrm{Cyo} /+; \mathrm{Tm} 3, \mathrm{Sb} / \mathrm{Tm} 6, \mathrm{~Tb}$ 
Two genotypes did display metastasis. One of these genotypes was homozygous for the IRER deficiency, and the other was heterozygous for the IRER deficiency.

1. ey1x-Gal4:UAS-DsRed/+ ; UAS-ScribRNAi, df(IRER)/df(IRER)

2. ey1x-Gal4:UAS-DsRed/+ ; UAS-ScribRNAi, df(IRER)/Tm3,Sb

The distributions for these two genotypes is illustrated in Figure 6.

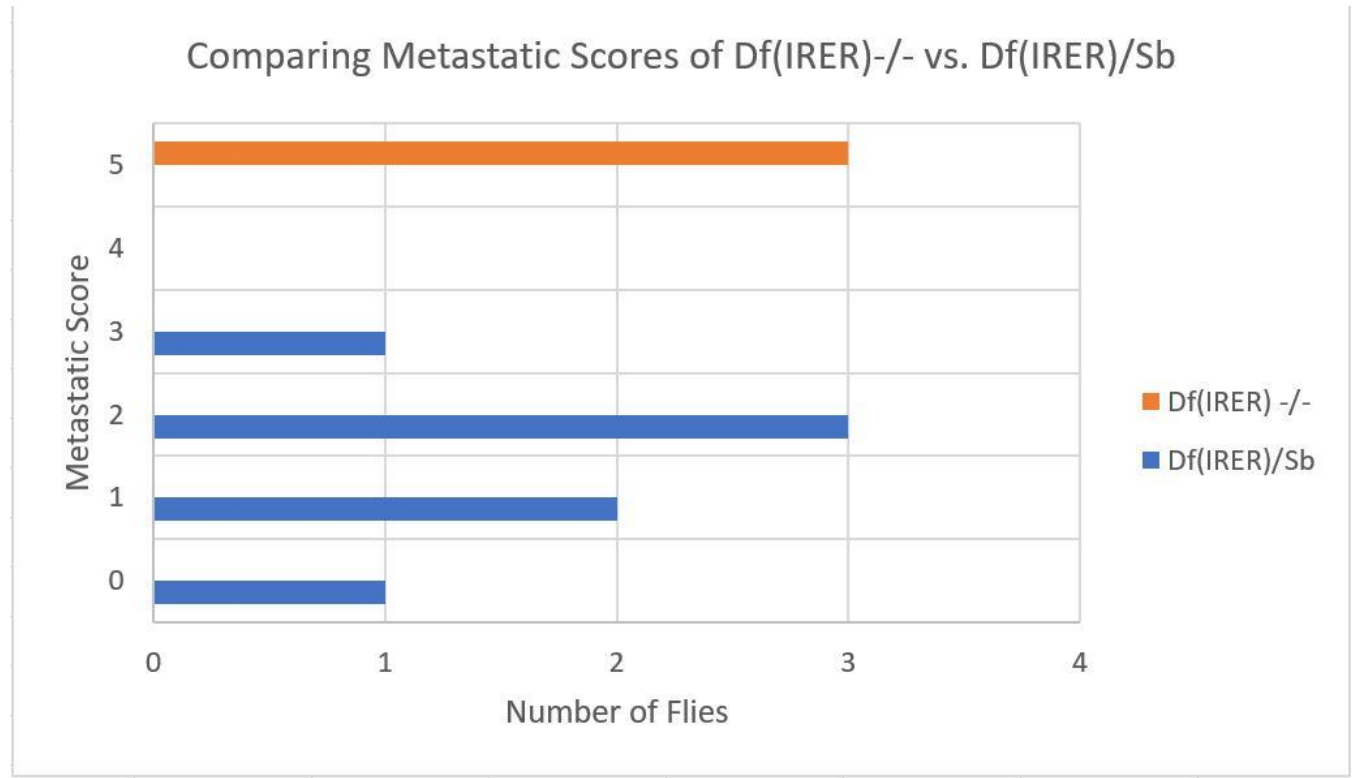

Figure 6. The distribution of metastatic scores for the ey1x-Gal4:UAS-DsRed/+ ; UAS-ScribRNAi, df(IRER)/df(IRER) and ey1x-Gal4:UAS-DsRed/+ ; UAS-ScribRNAi, df(IRER)/Tm3,Sb genotypes

The results from this cross showed that a homozygous IRER deficiency caused severe metastasis in $100 \%$ of the flies that managed to survive, as we only collected three throughout the entirety of our cross. This cross also gave us data on the heterozygous IRER deficiency. As we expected, a homozygous IRER deficiency caused more severe metastasis than a heterozygous IRER deficiency.

\section{Two Chromosomes Subjected to CRISPR-Cas9 Genome Editing Increased Metastasis}

In our first cross (1.1) we found that out of the four genotypes that were expressed, three had a metastatic score of zero. These three genotypes were:

1. ey1xGa14:UAS-DsRed/+ ; rpr BSKO (R8-1)/Tm6,Tb

2. Cyo/+ ; UAS-ScribRNAi, df(IRER)/rpr BSKO (R8-1)

3. Cyo/+ ; rpr BSKO (R8-1)/Tm6,Tb 
The genotype that did display metastasis was ey1xGal4:UAS-DsRed/+ ; UAS-ScribRNAi, df(IRER)/rpr BSKO (R8-1), as we had expected. We observed severe metastasis in the population, with $39.3 \%$ displaying a metastatic score of five.

In our second cross (1.2) we found that out of the four genotypes that were expressed, three had a metastatic score of zero. These three genotypes were:

1. ey1xGal4:UAS-DsRed/+ ; rpr BSKO (R15-1)/Tm6,Tb

2. Cyo/+ ; UAS-ScribRNAi, df(IRER)/rpr BSKO (R15-1)

3. Cyo/+ ; rpr BSKO (R15-1)/Tm6,Tb

The genotype that did display metastasis was ey1xGal4:UAS-DsRed/+ ; UAS-ScribRNAi, $\mathrm{df}(\mathrm{IRER}) / \mathrm{BSKO}$ (R15-1). This was unexpected and surprising, as there is no p53 binding site deletion in these flies. The metastatic distributions for crosses 1.1 and 1.2 is illustrated in Figure 7.

\section{Comparing Metastatic Scores of BSKO(R8-1)/Df(IRER)} vs. BSKO(R15-1)/Df(IRER)

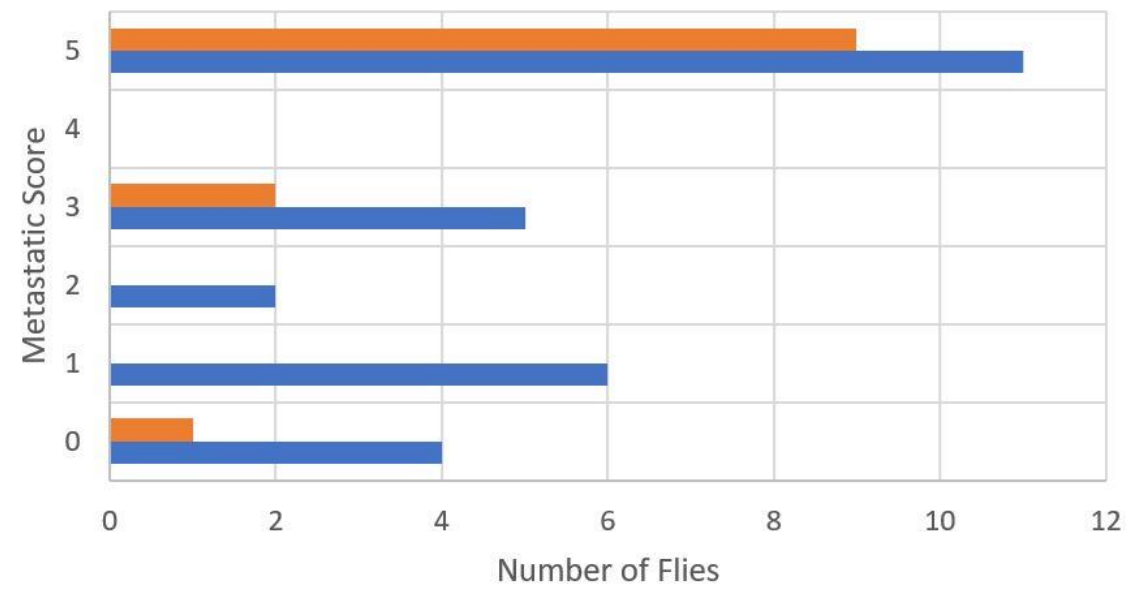

Figure 7: The distribution of metastatic scores for the ey1xGal4:UAS-DsRed/+ ; UAS-ScribRNAi, df(IRER)/rpr BSKO (R8-1) and ey1xGal4:UAS-DsRed/+ ; UAS-ScribRNAi, df(IRER)/BSKO (R15-1) genotypes.

Initially, the data from cross 1.2 seemed as though it would indicate that a heterozygous IRER deficiency was enough to cause significant metastasis, with $75 \%$ of the population displaying a metastatic score of five. However, data from our third cross must also be taken into account before drawing this conclusion.

Comparing cross 1.2 to cross 2 , it was clear that although both had a genotype that was heterozygous for an IRER deficiency, there was a significant difference in the severity of 
metastasis. Furthermore, on comparing cross 1.1 to cross 2 , it was shown that the deletion of the p53 binding motif was significant enough to cause more severe metastasis than just a heterozygous IRER deficiency.

\section{Discussion}

Our results from cross 2 replicated previous findings and confirmed that homozygous IRER deletion promotes metastasis. IRER is required for stress induced cell death. It is possible that increased survival of cells with scribble knocked down led to increased neoplasia and metastasis.

Our other two crosses (1.1 and 1.2) showed an intermediate phenotype between the homozygous and heterozygous IRER deficiency. Cross 1.1 contained a chromosome with the p53 binding motif deleted. Comparing cross 1.1 to cross 2 , we observed that metastasis was more severe in cross 1.1. This would indicate that the chromosome we tested did not have the potential to exacerbate the metastatic phenotype. However, we cannot draw this conclusion as cross 1.2 also displayed metastasis that was more severe than cross 2 , though it was a similar heterozygous IRER deficient genotype.

We have discussed two possibilities that could explain this data. One common concern for CRISPR-Cas9 genome editing is off target deletion. If CRISPR did attack other binding sites we would have to sequence the whole fly's genome to be sure, which would take some time. Another possibility is simply the genetic background of the flies we used in crosses 1.1 and 1.2 contributing to the metastatic phenotype. It is possible that the chromosome used in this fly line could have another defect, which may reveal new interesting findings.

Our next step will be to perform another metastasis cross with new controls that we can compare to the results from crosses 1.1 and 1.2. Hopefully, this cross will be able to definitively tell us whether the results we obtained from crosses 1.1 and 1.2 were due to a mistake in genome editing, or if the genetic background of the flies we used contributed to the metastasis we observed.

\section{Conclusion}

Cross 2 confirmed that homozygous IRER deletion exacerbates metastasis. Our other two experimental crosses both showed a phenotype that existed in between the homozygous and heterozygous IRER deficiency. The average metastatic score for the flies with the homozygous IRER deletion was 5, and the average metastatic score for the flies with the heterozygous IRER deletion was 1.57 . In cross 1.1 , the average metastatic score of the affected flies was 2.86 , while 
the average metastatic score of the affected flies in cross 1.2 was 4.25 . Comparing the cross 1.1 to cross 2, we can see that the metastasis is more severe in the BSKO(R8-1)/Df(IRER) flies compared to $\mathrm{Df}(\mathrm{IRER}) / \mathrm{Sb}$, which would indicate that the chromosome we tested does have the potential to exacerbate the metastatic phenotype. However, cross 1.2 unexpectedly displayed a more severe metastatic phenotype than the $\mathrm{Df}(\mathrm{IRER}) / \mathrm{Sb}$ genotype, which results in our data being inconclusive in regards to the impact of the p53 binding motif on limiting metastasis through apoptosis.

\section{References}

Altschul, S.F., Gish, W., Miller, W., Myers, E.W. \& Lipman, D.J. (1990). Basic local alignment search tool. Journal of Molecular Biology, 215. 403-410.

Amundson, S. A., Myers, T. G., \& Fornace, A. J., Jr. (1998). Roles for p53 in growth arrest and apoptosis: Putting on the brakes after genotoxic stress. Oncogene, 17. 3287-3299.

Busson D., Pret AM. (2007) GAL4/UAS Targeted Gene Expression for Studying Drosophila Hedgehog Signaling. In: Horabin J.I. (eds) Hedgehog Signaling Protocols. Methods of Inmolecular Biology ${ }^{\mathrm{TM}}$, 397. Humana Press

Duffy, M. J., Synnott, N. C., \& Crown, J. (2017). Mutant p53 As a Target For Cancer Treatment. European Journal of Cancer, 83. 258-265. doi.org/10.1016/j.ejca.2017.06.023

Mishra, A., Brat, D. J., \& Verma, M. (2014). P53 Tumor Suppression Network in Cancer Epigenetics. Methods in Molecular Biology Cancer Epigenetics, 597-605. doi:10.1007/978-1-4939-1804-1_31

Moore, C. B., Guthrie, E. H., Huang, M. T., \& Taxman, D. J. (2010). Short hairpin RNA (shRNA): design, delivery, and assessment of gene knockdown. Methods in molecular biology (Clifton, N.J.), 629, 141-158. doi:10.1007/978-1-60761-657-3_10

Powell, E., Piwnica-Worms, D., \& Piwnica-Worms, H. (2014). Contribution of p53 to Metastasis. Cancer Discovery, 4(4). doi:10.1158/2159-8290

Seyfried, T. N., \& Huysentruyt, L. C. (2013). On the origin of cancer metastasis. Critical reviews in oncogenesis, 18(1-2), 43-73.

Thurmond J, Goodman JL, Strelets VB, Attrill H, Gramates LS, Marygold SJ, Matthews BB, Millburn M, Antonazzo G, Trovisco V, Kaufman TC, Calvi BR and the FlyBase Consortium. (2019) FlyBase 2.0: the next generation. Nucleic Acids Res. 47(D1) D759-D765

Verma M., Kulshrestha S., Puri A. (2017) Genome Sequencing. In: Keith J. (eds) Bioinformatics. Methods in Molecular Biology, 1525. Humana Press, New York, NY

Zhang, C., Casas-Tintó, S., Li, G., Lin, N., Chung, M., Moreno, E., Moberg, K. H., Zhou, L. (2014). An intergenic regulatory region mediates Drosophila Myc-induced apoptosis and blocks tissue hyperplasia. Oncogene, 34(18). 2385-97. 
Zhang, Y., Lin, N., Carroll, P. M., Chan, G., Guan, B., Xiao, H., Yao, B., Wu, S. S., Zhou, L. (2008). Epigenetic blocking of an enhancer region controls irradiation-induced proapoptotic gene expression in Drosophila embryos. Developmental cell, 14(4). 481-93.

Zhao, Dongdong \& Feng, Xu \& Zhu, Xinna \& Tao, Wu \& Zhang, Xueli \& Bi, Changhao. (2017). CRISPR/Cas9-assisted gRNA-free one-step genome editing with no sequence limitations and improved targeting efficiency. Scientific Reports, 7. 10.1038/s41598-017-16998-8. 